\title{
Surgery compared to stereotactic body radiation therapy for early- stage non-small cell lung cancer: better, equivalent or worse?
}

\author{
Paul E. Van Schil \\ Department of Thoracic and Vascular Surgery, Antwerp University Hospital and Antwerp University, Edegem, Belgium \\ Correspondence to: Paul E. Van Schil, MD, PhD. Department of Thoracic and Vascular Surgery, Antwerp University Hospital and Antwerp University, \\ Wilrijkstraat 10, B-2650 Edegem (Antwerp), Belgium. Email: paul.van.schil@uza.be. \\ Provenance: This is an invited Editorial commissioned by the Section Editor Laura Chiara Guglielmetti (Cantonal Hospital Winterthur, Kantonsspital \\ Winterthur, Switzerland). \\ Comment on: Yu XJ, Dai WR, Xu Y. Survival Outcome after Stereotactic Body Radiation Therapy and Surgery for Early Stage Non-Small Cell Lung \\ Cancer: A Meta-Analysis. J Invest Surg 2017. [Epub ahead of print].
}

Submitted Sep 25, 2017. Accepted for publication Sep 27, 2017.

doi: $10.21037 /$ jtd.2017.10.36

View this article at: http://dx.doi.org/10.21037/jtd.2017.10.36

Surgical resection of early-stage non-small cell lung cancer (NSCLC) yields good long-term results on the condition that a complete $\mathrm{R} 0$ resection is performed according to the definition elaborated by a task force of the International Association for the Study of Lung Cancer (IASLC) (1). In 1995, after the study of the Lung Cancer Study Group (LCSG), which is the only phase III randomized controlled trial that has been published as full paper until now, lobectomy became the minimal resection and standard therapy for early-stage NSCLC $(2,3)$. Sublobar resection, especially wedge resection, was only accepted for compromised patients with a high cardiopulmonary comorbidity precluding a formal lobectomy. This changed with the new millennium and sublobar resection emerged as a potentially valid oncological treatment for very earlystage NSCLC, especially for pre-invasive or minimally invasive lesions (4). At the same time, similar to stereotactic brain irradiation, newer radiotherapy techniques allowed to precisely deliver a high dose of radiotherapy to smaller, peripherally located lung lesions, so-called stereotactic body radiation therapy or SBRT (5). The confusing term stereotactic radiosurgery should not be used as it does not include any surgical therapy. Phase II studies of SBRT for lung lesions demonstrated excellent ablation with encouraging local control during follow-up (5). In this way, SBRT became an alternative treatment to surgery in patients presenting a high risk for any surgical intervention. Over the recent years, the question arose whether SBRT could provide similar results as complete resection for early-stage
NSCLC in functionally operable patients. This topic is currently hotly debated at major oncological meetings.

In a recent, timely meta-analysis the authors examined this question in more detail and studied the efficacy of SBRT and surgery for early-stage NSCLC (6). They collected a total of 339 studies published between 2011 and 2017, of which 12 were included in the final analysis. Overall and disease-free survivals were compared between SBRT and surgery, as well as local control, regional and distant relapse. Their aggregate analysis showed that there was a significant benefit for SBRT regarding overall survival with a hazard ratio (HR) of 2.22, disease-free survival ( $\mathrm{HR}=2.37$ ), and distant recurrence $(\mathrm{HR}=1.77)$ with less recurrences in the SBRT group (6). In contrast, local and regional relapse occurred more frequently in the SBRT arm with a HR of 4.13. In the abstract the general conclusions of the authors were that more favorable outcomes are obtained with SBRT, and that surgery has no obvious advantages. However, in their conclusions at the end of manuscript they were more moderate after considering the limitations of their study. In this paragraph on page 5 they state that the choice of SBRT versus surgery might be best made at the individual patient level, and that all patients should be informed about the advantages and disadvantages of surgery and SBRT (6).

How to interpret this meta-analysis and its main conclusions? First of all, the authors admit several shortcomings in their review, mainly based on the fact that all included studies were retrospective with their inherent selection bias. Moreover, there are several inconsistencies, 
inaccuracies and confusing definitions in their manuscript. In the abstract they mention that finally, 14 studies were included, in Figure 1, 11 studies are mentioned and on page 3 in the first paragraph only 9 studies. In contrast, Table 1 lists 12 studies which seem to be the correct number. In the abstract the authors indicate that SBRT achieved inferior results compared with surgery, while they clearly mean superior for most of the analyzed parameters, as shown in the results section. Some unusual terms are used, e.g., "local control survival" and "regional control survival", while local and regional control are separate entities which should be clearly separated from survival data. On page 3 of the manuscript they mention several times in their analysis "randomized controlled trials" (RCT), while all included studies were retrospective and none was randomized. A critical analysis of included studies is not provided. At the start of the discussion on page 3 the authors state that lobectomy with sampling or dissection of mediastinal lymph nodes is standard therapy. To comply with the criteria for a complete R0 resection as mentioned above, a systematic nodal dissection including hilar and mediastinal lymph node stations, is required for every invasive NSCLC that undergoes surgical resection (1).

As discussed at major conferences, in general, several critical issues exist when comparing surgery to SBRT. These are not always adequately addressed in comparative papers or systematic reviews, and even in meta-analyses as the present study $(7,8)$.

In surgical series a clear documentation is provided of histology of the primary tumor and locoregional lymph nodes to define an exact pTNM and p-stage category. This is not the case for SBRT where only a clinical TNM stage can be given. Moreover, in SBRT series histology is not always obtained or required. In some studies this figure may even amount to $70 \%$ of the included patients having no pathological diagnosis (9). So, it is not always clear what exactly is irradiated and in some patients this might be a benign lesion. Precise lymph node staging is not always performed with SBRT and minimally invasive or invasive staging techniques are not often utilized. As there is a significant discrepancy between clinical and pathological staging, some patients may be understaged. In operated patients with $\mathrm{N} 1$ or $\mathrm{N} 2$ involvement adjuvant chemotherapy is given, and in specific subgroups even concurrent chemoradiotherapy to improve long-term outcome and reduce the risk of local and distant recurrence. Although the survival difference may be rather modest, it concerns a fairly large group of patients and the question arises how to select these patients after SBRT. For this reason some authors advocate the same rigorous lymph node evaluation as for a surgical candidate (10).

When considering SBRT for smaller lung lesions no uniform dose schedule is available that is universally agreed upon. A steep dose-response relationship is present (11). Respiratory movements make it difficult to apply the correct dose, especially for lesions located close to the diaphragm for which special techniques are required as breath-hold techniques, abdominal compression, respiratory tracking and respiratory gating (12).

Response evaluation after SBRT is quite troublesome due to the fact that inflammation is invariably present around the primary tumor. In fact, this may even increase the size and volume of the treated lesions shortly after radiotherapy administration. Specific criteria have been proposed, as the modified Response Criteria in Solid Tumors (RECIST), but currently, these are not validated (11). No precise definitions for local evaluation are available and in most radiotherapy series local control is defined as "absence of progressive disease”. In contrast, after complete surgical resection nothing remains of the primary tumor and draining lymph nodes, making it easier to detect local recurrent disease at an early stage. So, it should be realized that different criteria are used to define local control which gives rise to a so-called comparison bias (7). When comparing SBRT and surgery, not only local control should be taken into account as specific endpoint, but also overall and disease-free survival. This necessitates a clear documentation of local, regional, and distant recurrences as well as the precise causes of death.

For smaller peripheral lesions SBRT should currently be compared to minimally invasive surgical procedures. In a matched comparative analysis of patients with proven NSCLC, 41 patients undergoing video-assisted thoracic surgical (VATS) lobectomy were compared to a similar group treated with SBRT (13). Overall, recurrence-free, and cancer-specific survivals were significantly better in the surgical group. Are the same results obtained in compromised patients? In another, also matched comparative analysis 53 patients presenting a high risk for lobectomy, underwent sublobar resection (14). They were compared to a similar group treated with SBRT. No difference in overall survival was found indicating that in patients with severe comorbidity SBRT may yield similar results as surgery. This specific subgroup currently represents the best candidates to be treated by SBRT.

Lastly, in case of recurrent local or locoregional disease surgeons may have to perform so-called "salvage surgery" 
which is quite a new entity in thoracic surgery (15). When only peripheral lung parenchyma has been irradiated, lobectomy is feasible in most cases although no large series are available at the present time (16).

Only properly designed, prospective randomized controlled trials will provide a more definite answer when comparing surgery to SBRT for treatment of early-stage NSCLC. Recent pooled data of two randomized trials which closed due to poor accrual, did not resolve this issue and gave rise to an intense discussion $(17,18)$. Thoracic surgeons should be involved from the start when designing these trials to provide precise definitions and obtain a correct comparison between these two treatment modalities. Also, comorbidity factors that will determine outcome should be taken into account as a considerable number of patients will not die of their stage I NSCLC, but of other cardiopulmonary disorders equally related to smoking habits. Only in this way, we will be able to construct universally accepted therapeutic algorithms, and define those specific subcategories of patients that are optimally treated by SBRT or surgery.

\section{Acknowledgements}

None.

\section{Footnote}

Conflicts of Interest: The author has no conflicts of interest to declare.

\section{References}

1. Rami-Porta R, Wittekind C, Goldstraw P, et al. Complete resection in lung cancer surgery: proposed definition. Lung Cancer 2005;49:25-33.

2. Ginsberg RJ, Rubinstein LV. Randomized trial of lobectomy versus limited resection for T1 N0 non-small cell lung cancer. Lung Cancer Study Group. Ann Thorac Surg 1995;60:615-22; discussion 622-3.

3. Lederle FA. Lobectomy versus limited resection in T1 N0 lung cancer. Ann Thorac Surg 1996;62:1249-50.

4. Van Schil PE, Asamura H, Rusch VW, et al. Surgical implications of the new IASLC/ATS/ERS adenocarcinoma classification. Eur Respir J 2012;39:478-86.

5. McCloskey P, Balduyck B, Van Schil PE, et al. Radical treatment of non-small cell lung cancer during the last 5 years. Eur J Cancer 2013;49:1555-64.

6. Yu XJ, Dai WR, Xu Y. Survival Outcome after Stereotactic Body Radiation Therapy and Surgery for Early Stage
Non-Small Cell Lung Cancer: A Meta-Analysis. J Invest Surg 2017. [Epub ahead of print].

7. Van Schil PE, Van Meerbeeck J. Surgery or radiotherapy for early-stage lung cancer--a potential comparison bias. Lancet Oncol 2013;14:e390.

8. Van Schil PE. Results of surgery for lung cancer compared with radiotherapy: do we speak the same language. J Thorac Oncol 2013;8:129-30.

9. Lagerwaard FJ, Haasbeek CJ, Smit EF, et al. Outcomes of risk-adapted fractionated stereotactic radiotherapy for stage I non-small-cell lung cancer. Int J Radiat Oncol Biol Phys 2008;70:685-92.

10. van den Berg LL, Klinkenberg TJ, Groen HJ, et al. Patterns of Recurrence and Survival after Surgery or Stereotactic Radiotherapy for Early Stage NSCLC. J Thorac Oncol 2015;10:826-31.

11. Pennathur A, Luketich JD, Heron DE, et al. Stereotactic radiosurgery for the treatment of stage I non-small cell lung cancer in high-risk patients. J Thorac Cardiovasc Surg 2009;137:597-604.

12. Cole AJ, Hanna GG, Jain S, et al. Motion management for radical radiotherapy in non-small cell lung cancer. Clin Oncol (R Coll Radiol) 2014;26:67-80.

13. Hamaji M, Chen F, Matsuo Y, et al. Video-assisted thoracoscopic lobectomy versus stereotactic radiotherapy for stage I lung cancer. Ann Thorac Surg 2015;99:1122-9.

14. Matsuo Y, Chen F, Hamaji M, et al. Comparison of long-term survival outcomes between stereotactic body radiotherapy and sublobar resection for stage I non-small-cell lung cancer in patients at high risk for lobectomy: A propensity score matching analysis. Eur J Cancer 2014;50:2932-8.

15. Van Breussegem A, Hendriks JM, Lauwers P, et al. Salvage surgery after high-dose radiotherapy. J Thorac Dis 2017;9:S193-S200.

16. Uramoto H. Current Topics on Salvage Thoracic Surgery in Patients with Primary Lung Cancer. Ann Thorac Cardiovasc Surg 2016;22:65-8.

17. Chang JY, Senan S, Paul MA, et al. Stereotactic ablative radiotherapy versus lobectomy for operable stage I nonsmall-cell lung cancer: a pooled analysis of two randomised trials. Lancet Oncol 2015;16:630-7.

18. Opitz I, Rocco G, Brunelli A, et al. Surgery versus SABR for resectable non-small-cell lung cancer. Lancet Oncol 2015;16:e372-3.

Cite this article as: Van Schil PE. Surgery compared to stereotactic body radiation therapy for early-stage non-small cell lung cancer: better, equivalent or worse? J Thorac Dis 2017;9(11):4230-4232. doi: 10.21037/jtd.2017.10.36 